\title{
FIRST EXPERIMENTS USING THE IMAGE FORESTING TRANSFORM (IFT) ALGORITHM FOR SEGMENTATION OF REMOTE SENSING IMAGERY
}

\author{
A. R. Soares ${ }^{\text {a }}$,T. S. Körting ${ }^{\text {a }}$, L. M. G. Fonseca ${ }^{a}$ \\ ${ }^{a}$ Image Processing Division, National Institute of Space Research, INPE, São José dos Campos, Brazil \\ (anderson.soares,thales.korting,leila.fonseca)@inpe.br
}

KEY WORDS: Image Segmentation, Image Foresting Transform, Multiresolution Segmentation, Segmentation Comparison

\begin{abstract}
:
Image segmentation is a traditional method in Remote Sensing and a fundamental problem in image processing applications. It has been widely used, especially with the emergence of the Geographic Object-Based Image Analysis (GEOBIA). The results of segmentation must create uniform areas, which must allow a simpler interpretation by the users and simpler representation for classification algorithms. Several algorithms were proposed through the years, using different approaches. One that is widely used in Remote Sensing applications is the Multiresolution algorithm, that is based on the region growing method. Other, which has great potential and is applied in other research areas, is available on the Image Foresting Transform (IFT) framework, which has several image operators developed primarily for medical images. The Watershed from Grayscale Marker operator uses an edge image to perform the segmentation, however, we propose an extension of the edge detection algorithm, by summing normalized gradients of each band. This work aims to evaluate and compare these two segmentation algorithms, by comparing their results through supervised segmentation from reference regions, that were defined manually by an expert user. Quality measures were evaluated by four metrics, that represent the positional adjustment based the center of gravity, intensities, size, and the amount of overlap between the segment created by the algorithms and the reference segment. We selected 21 objects of a WorldView-2 multispectral image that were used to compute the metrics. Both methods reached similar results, by comparing the aforementioned 4 metrics applied to the 21 reference regions, IFT achieved better results for majority of regions. The IFT generated segments with similar shape when compared with the references, and the multiresolution generated results with similar sizes and positional adjustments. It may be explained by the fact that IFT uses an edge image to perform the segmentation. Both algorithms obtained similar agreement for intensity.
\end{abstract}

\section{INTRODUCTION}

Image segmentation is a fundamental problem in image processing, (Soille, 1999) defined segmentation as a process to split an image grouping the pixels by a similar attribute, such as the grey level, so the line which splits the areas, ideally, must be an edge. According to (Körting, 2012) the segmentation is one of the most challenging tasks in digital image processing.

Geographic Object-Based Image Analysis (GEOBIA) is devoted to developing automated methods to partition Remote Sensing imagery into meaningful image-objects, and assessing their characteristics through spatial, spectral and temporal scales (Hay and Castilla, 2008). It became widely used because it offers the potential to exploit geographical information.

The development of GEOBIA required a search for new methods for image segmentation. During the last decades, numerous techniques have been developed and applied in Remote Sensing analysis (Bins et al., 1996, Hay et al., 2005). The image segmentation algorithm that generates appropriate results has been the Multiresolution Segmentation (MRS) (Baatz and Schäpe, 2000), which probably is the most popular algorithm applied in GEOBIA.

Besides MRS good results, the search for new algorithms for image segmentation is still a necessity, and can not be limited by the MRS popularity. Different approaches may, also, produce similar results or even better results.

The Image Foresting Transform is a general tool for the design, implementation, and evaluation of image processing operators

\footnotetext{
${ }^{*}$ Corresponding author
}

based on connectivity (Falcão et al., 2004), it defines a minimumcost path forest in a graph, whose nodes are the image pixels and whose arcs are defined by an adjacency relation between pixels. The cost of a path is determined by a path-cost function $f$, which usually depends on local image properties (color, gradient, or pixel position) along the path between the nodes and the root.

The IFT Watershed from Grayscale Marker (called Watergray from this point), is one IFT operator in which the segmentation is computed from a gray scale image. According (Lotufo et al., 2002) it puts together several steps used in classical watershed morphological segmentation strategies in a single algorithm.

This paper evaluates and compares the Watergray operator, adapted to the Remote Sensing context, with the MRS algorithm.

\section{METHODOLOGY}

We compared the algorithms using 4 quality measures applied in 21 selected regions (Figure 1), on a WorldView-2 Multispectral image.

As the Watergray uses an edge image to perform the segmentation we implemented on MATLAB an adaptation of the traditional way to compute the gradient of the image. As described in Equation 1 we computed the traditional gradient for each band and normalized each one. With this preprocessing we expect that each band has the same importance when all gradients are summed.

$$
\nabla_{\text {image }}=\sum_{i}^{N} w_{N} * \nabla_{N}
$$




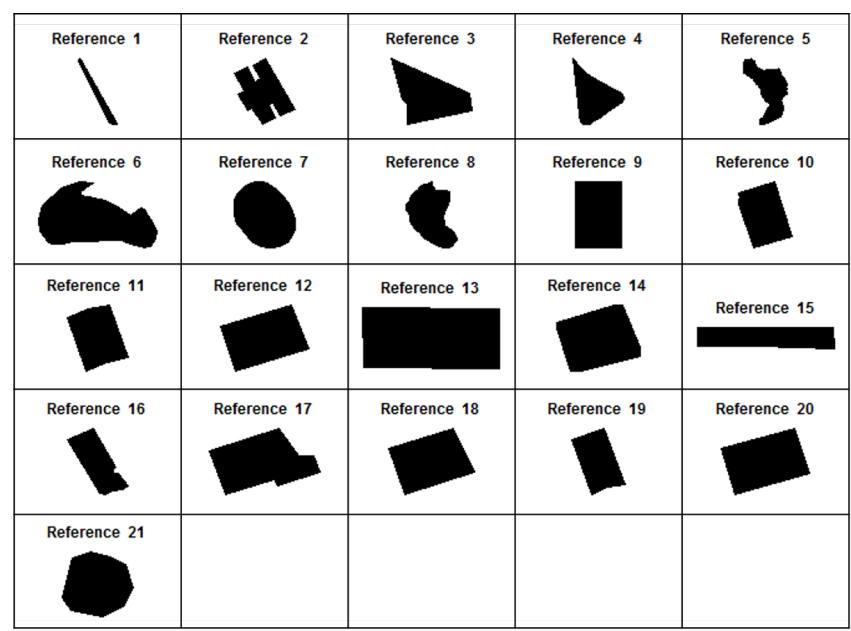

Figure 1. Rerefence regions.

In comparison with MRS algorithm, by using our approach it is possible to create a gradient image with different weights for each band. This way, we can enhance some feature or object, considering its spectral characteristics.

Since Watergray is not able to handle georeferenced images, we developed an algorithm in C++ using the TerraLib library (Câmara et al., 2008), to project the labeled image provided by Watergray to the same reference system of the WorldView-2 image.

To perform the experiment we used the following tuning parameters for MRS algorithm: 45 scale, 0.3 shape e 0.7 compactness, and weight 2 for infrared bands and 1 for other bands. For the Watergray the cost applied was 12 and weight 2 for infrared bands and 1 for the other bands.

For evaluation of the segmentation algorithms we applied a supervised approach, as proposed by (Delves et al., 1992). Each region of the reference segmentation is selected to be evaluated in the comparison. Consider a reference segmentation with $K$ segments and the evaluated segmentation with $L$ segments, both with $W$ columns and $H$ rows, a given region $i$ in the reference segmentation, and a given object in the segmentation being evaluated, $f$. The $\left\langle g_{i}>\right.$ notation represents the average of the measure $g$ in a given region $i, N(i)$ represents the number of pixels of $i$ and $x_{i}$ and $y_{i}$ indicate the location of some pixel inside $i$ region, respectively the column and row (Reis et al., 2015). Two matrices, $G f$ and Fit, both with $H$ rows and $W$ columns, are constructed using the following equations:

$$
\begin{gathered}
G f(i, f)=\frac{N(i \bigcap f)}{N(i \bigcup f)} \\
F i t=\frac{x d+y d+\frac{p d+i d}{2}}{G f(i, f)}
\end{gathered}
$$

in which:

$$
\begin{aligned}
& x d=\frac{\left|<x_{i}>-<x_{f}\right\rangle \mid}{H} \\
& y d=\frac{\left|<y_{i}>-<y_{f}>\right|}{W}
\end{aligned}
$$

Table 1. Average and Standard Deviation of Metrics

\begin{tabular}{c|c|c|c|c} 
& \multicolumn{2}{|c|}{ MRS } & \multicolumn{2}{c}{ Watergray } \\
\hline Metric & Average & Std. & Average & Std. \\
\hline FIT XY & 0.8993 & 0.1170 & 0.8821 & 0.1376 \\
FITI & 0.6086 & 0.1966 & 0.6146 & 0.1840 \\
Gshape & 0.6491 & 0.1804 & 0.6632 & 0.1836 \\
FIT N & 0.5555 & 0.2105 & 0.5390 & 0.2210 \\
FIT M & 0.6781 & 0.0750 & 0.6738 & 0.0843
\end{tabular}

$$
\begin{gathered}
p d=\frac{|N(i)-N(f)|}{|N(i)+N(f)|} \\
i d=\frac{|<V(i)>-<V(f)>|}{|<V(i)>+<V(f)>|}
\end{gathered}
$$

With those matrices, this approach computes 4 metrics: FIT XY, FITI, FITN, and Gshape. Each measure is calculated for each region considering a reference, that were defined manually by an expert user.

The metric FITXY, Equation 8, evaluates the displacement between the center of gravity of polygon generated by segmentation algorithms and the reference polygon. FITI, Equation 9, checks the grey level intensity of the segments, using for that, the original image.

The FITN, Equation 10, assesses the similarity between the size of the reference polygon and the size of the segment generated by the analyzed algorithms. Gshape, Equation 11, evaluates the shape similarity between the segments generated by algorithms and the reference.

$$
\begin{gathered}
F I T X Y=1-\frac{x d+y d}{2} \\
F I T I=1-\frac{|<V(i)>-<V(f)>|}{<V(i)>+<V(f)>} \\
F I T N=1-\frac{|N(i)-N(f)|}{N(i)+N(f)} \\
\text { Gshape }(i, f)=\frac{N(i \bigcap f)}{N(i \bigcup f)}
\end{gathered}
$$

All metrics produce values in the interval $[0,1]$, with 1 being the best. In order to merge all metrics in a single value, we computed also the average value of the aforementioned metrics, and it was denoted by FITM.

\section{PRELIMINARY RESULTS}

Table 1 shows the average obtained by each metric analyzed for both algorithms in all 21 reference regions. By analyzing this table we can observe that Watergray achieved better results for Gshape metric, while the MRS obtained better results for FITN and FITXY. For the other metrics, the results were similar.

\subsection{Positional adjustment - FITXY}

As can be observed on Figure 2 the MRS and Watergray generated similar segments, however, some segments generated by Watergray were slightly shifted, the difference was subtle. 


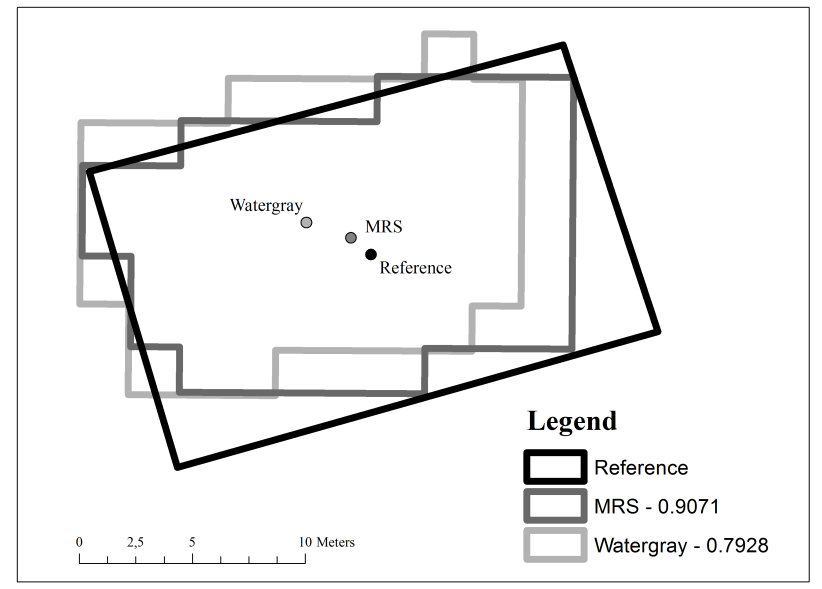

Figure 2. Center of gravity of segments - The FITXY metric.

\subsection{Intensities - FITI}

Again both algorithms produced similar responses, the difference of averages was below 0.001 . However, it is important to address that the standard deviation for this metric was high for both algorithms (around 0.19).

\subsection{Overlap - Gshape}

For this metric the Watergray had slightly better results, around 0.02 , this can be explained by the edge image used by Watergray, that allowed a better definition of the objects. Both algorithms had a high standard deviation, around 0.18 .

\subsection{Size - FITN}

For this metric the MRS naturally has an advantage, since it has a specific parameter that influences on the average size of resultant polygons, while the Watergray does not have such capability. In some cases the Watergray generated segments smaller than the references, or created some holes on the segment. Also, it is important to call attention to the fact that this metric had the worst results for both algorithms, and also the highest standard deviation, around 0.21 for MRS and 0.22 for Watergray.

\subsection{Average - FITM}

As aforementioned, the FITM is obtained by the average of the 4 metrics, therefore, it provides a global understanding of the similarity between the reference and the segment generated by algorithms. For most of the polygons the differences were small, around 0.004 . Therefore, the results indicate a good agreement between the algorithms and the analyzed polygons.

\subsection{Regularity}

We also evaluated if the regularity of the objects influences on the performance of the algorithms. From the 21 analyzed polygons, 13 were regular, while 8 had irregular shapes. Both algorithms obtained better results for the irregular polygons in almost all metrics, only the Gshape metric had poor results, around 0.59 . The FITI metric had the major gain, almost 0.4 for both algorithms. The small advantage obtained by MRS on the FITN and FITXY metrics, when all polygons were analyzed together, persisted only for the regular objects.

\section{CONCLUSION}

In this paper we evaluated and compared the Watergray and the MRS algorithms for Remote Sensing image segmentation. The Watergray had better results for the Gshape metric, however, it did not obtain as good results for FITN as MRS. Both algorithms obtained similar agreement for FITXY and FITI.

Those results indicate that both algorithms produced equivalent segmentations, however, new tests must be conducted to evaluate the Watergray on images of different domains and spatial resolutions. We also conclude from the results that Watergray has a great potential to segmentation of Remote Sensing imagery. For further studies, we will adapt the Watergray to handle small polygons and implement it on the TerraLib library (Câmara et al., 2008).

\section{REFERENCES}

Baatz, M. and Schäpe, A., 2000. Multiresolution segmentation: an optimization approach for high quality multi-scale image segmentation. In: Wichmann-Verlag (ed.), XII Angewandte Geographische Informationsverarbeitung, Vol. pp, Herbert Wichmann Verlag, Heidelberg, pp. 12-23.

Bins, L., Fonseca, L., Erthal, G. and Ii, F., 1996. Satellite imagery segmentation: a region growing approach. Simpósio Brasileiro de Sensoriamento Remoto 8, pp. 677-680.

Câmara, G., Vinhas, L., Ferreira, K., Queiroz, G., Souza, R., Monteiro, A., Carvalho, M., Casanova, M. and Freitas, U., 2008. TerraLib: An open source GIS library for large-scale environmental and socio-economic applications. Open Source Approaches in Spatial Data Handling 2, pp. 247-270.

Delves, L., Wilkinson, R., Oliver, C. and White, R., 1992. Comparing the performance of sar image segmentation algorithms. International Journal of Remote Sensing 13(11), pp. 2121-2149.

Falcão, A., Stolfi, J. and de Alencar Lotufo, R., 2004. The image foresting transform: theory, algorithms, and applications. IEEE Transactions on Pattern Analysis and Machine Intelligence 26(1), pp. 19-29.

Hay, G. and Castilla, G., 2008. Geographic Object-Based Image Analysis (GEOBIA): A new name for a new discipline. In: T. Blaschke, S. Lang and G. Hay (eds), Object-Based Image Analysis: Spatial Concepts for Knowledge-Driven Remote Sensing Applications, Springer-Verlag, Berlin, Heidelberg, chapter 1.4 , pp. $75-89$.

Hay, G. J., Castilla, G., Wulder, M. A. and Ruiz, J. R., 2005. An automated object-based approach for the multiscale image segmentation of forest scenes. International Journal of Applied Earth Observation and Geoinformation 7(4), pp. 339-359.

Körting, T., 2012. GeoDMA: A toolbox for data mining, object-based and multi-temporal analysis of remote sensing imagery. PhD thesis, Brazil's National Institute for Space Research (INPE).

Lotufo, R. A., O, A. X. F. and Zampirolli, F. A., 2002. IFTWatershed From Gray-Scale Marker. In: SIBGRAPI'02, pp. 146-152.

Reis, M. S., de Oliveira, M. A. F., Korting, T. S., Pantaleo, E., Sant'Anna, S. J. S., Dutra, L. V. and Lu, D., 2015. Image segmentation algorithms comparison. In: 2015 IEEE International Geoscience and Remote Sensing Symposium (IGARSS), pp. 43404343.

Soille, P., 1999. Morphological image processing: Principles and Applications. Cambridge University Press. 